doi: 10.2306/scienceasia1513-1874.2013.39.111

\title{
A taxonomic revision of the genus Eragrostis in Thailand
}

\author{
Wanwipha Chaisongkram $^{\mathrm{a}}$, Pranom Chantaranothai ${ }^{\mathrm{a}, *}$, Trevor R. Hodkinson ${ }^{\mathrm{b}}$ \\ a Applied Taxonomic Research Centre, Department of Biology, Faculty of Science, Khon Kaen University, \\ Khon Kaen 40002 Thailand \\ b School of Natural Sciences, Trinity College Dublin, Dublin 2, Ireland \\ ${ }^{*}$ Corresponding author, e-mail: pranom@kku.ac.th
}

Received 20 Jun 2012

Accepted 5 Feb 2013

\begin{abstract}
A taxonomic account of Eragrostis in Thailand is provided, in which 23 species are recognized. An artificial key to species and species descriptions is provided along with distributional and ecological data for all taxa. The taxonomic status of several Thai Eragrostis taxa is updated. Four species: E. alopecuroides, E. malayana, E. spartinoides, and E. zeylanica are reduced to synonymies of E. ciliata, E. montana, E. brownii, and E. brownii, respectively, and three species, E. ciliaris, E. cylindrica, and E. tef, are reported to occur in Thailand for the first time.
\end{abstract}

KEYWORDS: Eragrostideae, love grass, Poaceae

\section{INTRODUCTION}

Eragrostis belongs to the family Poaceae, tribe Eragrostideae $^{1}$. It is recognized as the largest genus within the subfamily Chloridoideae ${ }^{2}$ with ca. 420 species $^{3}$. It is widely distributed in tropical to temperate regions of the world and is found in open habitats and sometimes forests from sea level to high altitudes $^{2,4,5}$. Eragrostis was first published by Wolf in $1776^{6}$ in "Genera Plantarum" while the type of the generic name was designated by Ross ${ }^{7}$ based on E. minor Host. The origin of the name is ambiguous since there is no evidence concerning the generic name when it was first described. However, "eros" is Greek for "loving in an erotic sense" and Agrostis is a common genus that superficially resembles some Eragrostis species.

The previous taxonomic treatment of Eragrostis in Thailand recognized 35 species ${ }^{8}$ but it was confined to only a species list with some synonymies, without providing a thorough taxonomic treatment. The present paper provides a comprehensive taxonomic account of Thai Eragrostis for the Flora of Thailand project including an artificial key to species, updated nomenclature, important synonymies, distribution data, ecological information, vernacular names, and notes for each species.

\section{MATERIALS AND METHODS}

The treatment in this study was based on newly collected material from the first author's field work and herbarium specimens housed in the following herbaria: $\mathrm{AAU}, \mathrm{BK}, \mathrm{BKF}, \mathrm{BM}, \mathrm{C}, \mathrm{CMU}, \mathrm{E}, \mathrm{G}, \mathrm{K}$,
KKU, L, LINN, QBG, and TCD (Herbarium abbreviations following Ref. 9). A large number of mature samples were used to provide species descriptions and key construction. Ecological and distributional data were collected for each species during the field work, and were recorded from the label information of existing vouchers. Measurements were taken using a stereo microscope (Nikon SMZ645) after softening by warm water. Synonyms given were based on close examination of accepted names and synonymy lists in the taxonomic literature of Thailand and neighbouring regions.

\section{RESULTS AND DISCUSSION}

\section{Taxonomic treatment}

Eragrostis Wolf, Gen. Pl.: 23 (1776). Type species: Eragrostis minor Host, Icon. Descr. Gram. Austriac., 4: 15 (1809). Lectotype designated by Ross, Acta Bot. Neerl. 15: 157 (1966).

Annual or perennial, sometimes rhizomatous, tufted. Culms erect or geniculately ascending, slightly compressed or terete. Leaf-sheaths loose, coriaceous to membranous. Auricles glabrous or hairy. Ligules membranous or a fringe of long hairs. Collars glabrous or a row of long hairs, sometimes glandular. Leaf-blades apex acute to acuminate or attenuate. Panicles open or contracted or spiciform. Spikelets laterally (all of ours) or dorsally compressed to terete, with many florets, the upper sterile or reduced; breaking up in various ways; rachilla disarticulating or persistent. Lower glumes 1-nerved, 1-keeled. Upper glumes 1-3-nerved, 1-keeled. Florets bisexual. 
Lemmas 3-nerved, keeled. Paleas boat-shaped, longitudinally bowed-out, 2-nerved, 2-keeled, margins infolded. Lodicules 2. Stamens 1-3. Caryopses with adherent pericarp.

1. Eragrostis atrovirens (Desf.) Trin. ex Steud., Nom. Bot. ed. 2, 1: 562 (1840). Poa atrovirens Desf., Fl. Atlant. 1: 73 (1798). Type: Algeria, Barberia, in arvis incutis prope La Calle, Desfontaines 160 (holotype FI, picture K!; isotypes BAA-1006, P).E. luzoniensis Steud., Syn. Pl. Glumac. 1: 266 (1854). Type: Philippines, Luzon, H. Cuming 1416 (holotype $\mathrm{P}$; isotypes BM!, E!, K, L). Fig. 1a.

Perennial, blue-green, glaucous. Culms 30$150 \mathrm{~cm}$ tall, erect or geniculately ascending. Leafsheaths $30-50 \mathrm{~cm}$ long, coriaceous with membranous margins, glabrous. Auricles bearded with hairs. Ligules ciliolate membranous. Collars glabrous. Leaf-blades $10-25 \mathrm{~cm}$ by $5-8 \mathrm{~mm}$, upper surface scabrous, lower surface glabrous. Panicles open, oblong or ovate, $30-50$ by $8-10 \mathrm{~cm}$, branches glabrous. Spikelets $8-10$ by $1-1.8 \mathrm{~mm}$, with $8-20$ fertile florets, lanceolate-oblong; florets falling from the base of spikelet upwards; rachilla persistent. Lower glumes $1-1.5 \mathrm{~mm}$ long, caducous, ovate to lanceolate, keel scabrous, apex acute. Upper glumes $1.5-1.8 \mathrm{~mm}$ long, caducous, lanceolate, 1-nerved, keel scabrous, apex acute. Lemmas 2-2.5 mm long, caducous, oblongelliptic, apex acute to subobtuse. Paleas $1.8-2 \mathrm{~mm}$ long, caducous, keels scaberulous, apex acute to obtuse. Stamens 3; anthers ca. $0.7 \mathrm{~mm}$ long. Caryopses 0.4-0.6 mm long, ellipsoid, terete, pericarp striate and reticulate, reddish brown.

Distribution.- North and South America, Europe, Africa, Asia, Australasia, and Pacific.

Ecology.- Common in mixed deciduous forest, often in swampy grassland.

Vernacular.- Ya krok khiao.

Notes. - This species is easily recognized by its blue-green and glaucous appearance. Veldkamp ${ }^{10}$ accepted E. luzoniensis as a species but the isotypes of this species housed in the BM and E matched well with $P$. atrovirens and the name $E$. atrovirens has priority.

Specimens examined.- Nan: Doi Phu Kha National Park, 6 September 2008, W. Chaisongkram 133 (KKU). Loei: Phu Kradueng National Park, 1300 m, 18 March 1958, Th. Sørensen, K. Larsen \& B. Hansen 2239 (C); 27 March 2006, W. Chaisongkram 40 (KKU). Nakhon Ratchasima: Bua Luang, 1100 m, 1 February 1964, B. Hansen, G. Seidenfaden \& T. Smitinand 11012 (BKF, C, E). Chanthaburi: Makham, 14 June 1963, K. Larsen 10106 (BKF); 8 November

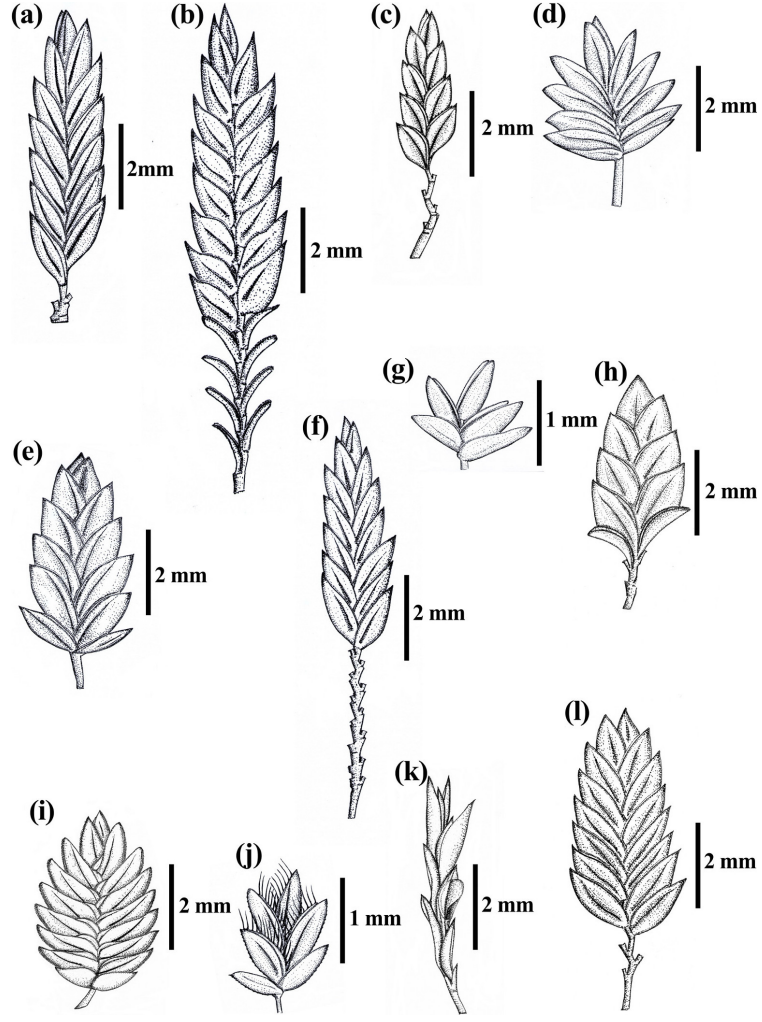

Fig. 1 Spikelet. (a) E. atrovirens with caducous paleas (below); (b) E. brownii with persistent paleas (below); (c) E. burmanica with zig-zag rachilla; (d) E. capensis; (e) E. cilianensis; (f) E. gangetica; (g) E. japonica with florets breaking up from above downwards on spikelet; (h) E. montana with straight rachilla; (i) E. superba; (j) E. tenella with tubercle-based hairs on palea keels; (k) E. tenuifolia with caryopsis adhering to palea; and (1) E. unioloides. All illustrations were drawn by Wanwipha Chaisongkram from W. Chaisongkram 133 (KKU), 136 (KKU) and 91 (KKU), Th. Sørensen, K. Larsen \& B. Hansen 2244 (BKF) and 4423 (BKF), W. Chaisongkram 193 (KKU), 106 (KKU) and 178 (KKU), S.N. (BKF), W. Chaisongkram $92(\mathrm{KKU}), 130(\mathrm{KKU})$ and $140(\mathrm{KKU})$, respectively.

2008, W. Chaisongkram 154 (KKU). Ranong: Klong Naka, 24 February 1974, R. Geesink, P. Hipko \& C. Charoenphol 7542 (BKF, C).

2. E. brownii (Kunth) Nees, Cat. Indian Pl.: 105 (1834). Poa brownii Kunth, Révis. Gramin. 1: 112 (1829). Lectotype (designated by Lazarides 1997) ${ }^{11}$ : Australia, Queensland, Port Curtis District, R. Brown 6284 (BM!).-E. zeylanica Nees \& Meyen, Gramineae: 72-73 (1841). Type: Sri Lanka, Macrae s.n. (holotype picture $\mathrm{K}$; isotype $\mathrm{BM}$ !). - Uniola spicata Llanos, Fragm. Pl. Filip.: 33 (1851), non L. 
Table 1 Key to the species.

1. Spikelet breaking up below glumes and falling as a whole spikelet, palea with winged keels, spikelet broadly elliptic to ovate, 3-4 mm wide

18. E. superba

1. Spikelet breaking up above glumes in various ways and not falling as a whole

2. Florets falling entire from the top of spikelet downwards

3. Palea keels ciliate, hairs $0.2-0.5 \mathrm{~mm}$ long

4. Panicle oblong; the lowermost branch, $2-2.5 \mathrm{~cm}$ long

4. Panicle ovate; the lowermost branch longest, $6-8 \mathrm{~cm}$ long

12. E. japonica

3. Palea keels with tubercle-based hairs, hairs $1-1.3 \mathrm{~mm}$ long

5. Pericarp rugulose; panicle open, $2-8 \mathrm{~cm}$ wide

6. Culm with sticky glandular patches below nodes

6. Culm glabrous or scaberulous below nodes

7. Spikelet lanceolate to oblong, $2-4 \mathrm{~mm}$ long

7. Spikelet ovate to ovate-oblong, delicate, $1.5-2 \mathrm{~mm}$ long

5. Pericarp finely striate; panicle spiciform or contracted, $1-2 \mathrm{~cm}$ wide

8. Inflorescence axis plumose

8. Inflorescence axis glabrous or scabrous

10. E. diplachnoides

2. Florets falling from the base of spikelet upwards

9. Caryopsis trigonous to reniform

10. Glumes subequal

11. Lemma caducous; spikelet dark purple

11. Lemma tardily breaking up; spikelet green

23. E. viscosa

17. E. riparia

20. E. tenella

7. E. ciliata

6. E. ciliaris

10. Glumes unequal, lower glume obviously smaller than the upper

12. Spikelet $3-4$ by $0.5-0.8 \mathrm{~mm}$; annuals; anther ca. $0.1 \mathrm{~mm}$ long

12. Spikelet $6-10$ by $1-1.5 \mathrm{~mm}$; perennials; anther ca. $0.5 \mathrm{~mm}$ long

9. Caryopsis ellipsoid or orbicular

13. Lemma and palea falling together or sometimes the palea falling after the lemma

14. Spikelet 1-1.8 mm wide, lanceolate-oblong, oblong or elliptic

15. Stamens 3

16. Spikelet $8-10 \mathrm{~mm}$ long; plant blue-green and glaucous

16. Spikelet 3-8 $\mathrm{mm}$ long with long pedicel; plant green

1. E. atrovirens

3. E. burmanica

15. Stamens 2

11. E. gangetica

14. Spikelet $2-4 \mathrm{~mm}$ wide, ovate or elliptic

17. Collar glabrous; spikelet normally pinkish

22. E. unioloides

17. Collar sparsely or densely pilose; spikelet pale brown sometimes tinged with blackish patches; plant with compacted dead sheaths at the base of culm

13. Lemma falling with caryopsis, palea persistent

4. E. capensis

18. Plant with crateriform glands

18. Plant without crateriform glands

19. Rachilla eventually breaking up from the top of spikelet downwards

20. Anther 0.4-0.6 mm long; perennials

14. E. nigra

19. E. tef

16. E. pilosa

21. E. tenuifolia

20. Anther $0.1-0.2 \mathrm{~mm}$ long; annuals

5. E. cilianensis

19. Rachilla persistent

21. Palea with slightly winged keels; caryopsis sub-globose

21. Palea keels without wing; caryopsis ellipsoid or orbicular

22. Caryopsis ellipsoid, terete

22. Caryopsis orbicular, slightly laterally compressed

2. E. brownii

8. E. cumingii

9. E. cylindrica

13. E. montana

15. E. nutans

Sp. Pl.: 71 (1753). Neotype (designated by Veldkamp 2002): Philippines, Luzon, E.D. Merrill Sp. Blancoan 170 (US; isoneotypes BM!, K!, L!, P).—E. spartinoides Steud. Syn. Pl. Glumac. 1: 265 (1854). Type: Philippines, Luzon, Laguna, Cuming 668 (holotype P; isotypes BM!, E!, G!, K!, L). Fig. 1 b.
Perennial. Culms $20-45 \mathrm{~cm}$ tall, erect to geniculately ascending. Leaf-sheaths $2-4 \mathrm{~cm}$ long, margins with tubercle-based hairs. Auricles bearded with long hairs. Ligules a fringe of hairs. Collars with a row of long hairs. Leaf-blades $5-15 \mathrm{~cm}$ by $2-4 \mathrm{~mm}$, both surfaces with sparse tubercle-based hairs. Panicles 
open, clustered or spiciform, ovate to oblong, 1530 by $1-5 \mathrm{~cm}$, bearded in axils. Spikelets $4-15$ by $0.8-1.5 \mathrm{~mm}$, with $10-60$ fertile florets, oblong; florets falling from the base of spikelet upwards, but rachilla ultimately breaking up from the top downwards; rachilla straight. L. glumes $1.2-1.6 \mathrm{~mm}$ long, caducous, lanceolate, keel scabrous, apex acuminate. Upper glumes $1.5-1.8 \mathrm{~mm}$ long, caducous, lanceolate, 1-nerved, keel scabrous, apex acuminate. Lemmas 2.7-3 mm long, caducous, ovate, scaberulous, membranous, apex acuminate. Paleas 2-2.2 mm long, persistent, keels ciliate, apex obtuse. Stamens 3; anthers 0.4-0.6 mm long. Caryopses 0.4-0.6 mm long, orbicular, slightly laterally compressed, pericarp finely reticulate, brown.

Distribution.- Europe, Asia, Australasia, and Pacific.

Ecology.- Common in dry deciduous forest and on open sandy soil.

Vernacular. - Ya wai.

Notes.- E. brownii is a variable species, especially in the size and the shape of its inflorescence. It has been confused with the annual species, E. cumingii, but it differs by having bigger anthers, 0.4-0.6 mm long. Veldkamp ${ }^{10}$ noted that this species has often been called E. elongata (Willd.) Jacq., species name which has been applied for the 3anthered species group in Southeast Asia and some of which are distributed from India to Australia, e.g., E. brownii, E. bulbillifera Steud., E. cumingii Steud., E. pubescens (R.Br.) Steud., E. spartinoides Steud., E. subsecunda (Lam.) E. Fourn. and E. zeylanica Nees. However, Shouliang and Peterson ${ }^{12}$ treated the 2-anthered species as E. elongata in the Flora of China. In Thailand, we have never found a specimen with two anthers.

Specimens examined-C Chiang Mai: Doi Suthep National Park, 450 m, 12 May 1958, Th. Sørensen, K. Larsen \& B. Hansen 3375 (BKF, C). Nong Khai: Phu Wua Wildlife Sanctuary, 26 October 2008, W. Chaisongkram 136 (KKU). Khon Kaen: Mueang Phon, 400 m, 23 March 1958, Th. Sørensen, K. Larsen \& B. Hansen 2468 (BKF, C, E); Khon Kaen University, 22 November 2009, W. Chaisongkram 198 (KKU). Nakhon Ratchasima: 29 December 1962, C. Phengkhlai 655 (BKF, C). Kanchanaburi: Chokgrading waterfall, 25 August 2009, W. Chaisongkram 189 (KKU). Saraburi: $15 \mathrm{~km}$ east of Saraburi, $150 \mathrm{~m}$, 12 March 1958, Th. Sørensen, K. Larsen \& B. Hansen 2073 (C). Trat: Ko Chang, 5 m, 19 February 1955, T. Smitinand 2242 (BKF). Chumphon: Pratew, 23 February 2008, W. Chaisongkram 128 (KKU). Surat Thani: Kanchanadit, 1.5 m, 1 August 1927, A.F.G.
Kerr 13060 (BM, K).

3. E. burmanica Bor, Kew Bull.: 166 (1951). Type: Myanmar, Prome District, fields, 20 Jul 1948, U. Thein Lwin 582 (holotype K!). Fig. 1c.

Perennial. Culms $20-40 \mathrm{~cm}$ tall, erect or slightly geniculate. Leaf-sheaths 4-7 cm long, chartaceous to membranous. Auricles bearded with long hairs. Ligule a fringe of hairs. Collars glabrous. Leafblades $7-15 \mathrm{~cm}$ by 3-6 $\mathrm{mm}$, upper surface sparsely hairy, lower surface glabrous. Panicles lax with long pedicels, $20-35$ by $8-12 \mathrm{~cm}$, pedicels compressed or triquetrous. Spikelets $3-8$ by $1-1.5 \mathrm{~mm}$, with $5-15$ fertile florets, elliptic; florets falling from the base of spikelet upwards; rachilla persistent. L. glumes 0.8-1 mm long, caducous, lanceolate to elliptic, keel scabrous, apex acuminate. Upper glumes $1.2-1.5 \mathrm{~mm}$ long, caducous, lanceolate, 1-nerved, keel scabrous, apex acuminate. Lemmas 1-1.4 mm long, caducous, ovate, scaberulous, membranous, apex acute to acuminate. Paleas 0.9-1.2 mm long, caducous, keels scaberulous, apex acute. Stamens 3; anthers ca. $0.4 \mathrm{~mm}$ long. Caryopses $0.4-0.5 \mathrm{~mm}$ long, ellipsoid, terete, pericarp finely reticulate, reddish brown.

Distribution.- Myanmar and Thailand.

Ecology.- Common in open areas and on sandy soils.

Vernacular. - Ya krok.

Notes. - This species is characterized by its lax panicle with long pedicels.

Specimens examined.- Phitsanulok: Thung Salaeng Luang National Park, 700 m, 21 July 1966, K. Larsen, T. Smitinand \& E. Warncke 671 (AAU, BKF). Phetchabun: Nam Nao National Park, 24 August 2006, W. Chaisongkram 91 (KKU). Chaiyaphum: Thung Kamang, 850 m, 2 June 1974, R. Geesink, T. Hattink \& C. Phengkhlai 7140 (BKF).

4. E. capensis (Thunb.) Trin., Mém. Acad. Imp. Sci. St.-Pétersbourg, Sér. 6, Sci. Math. 1(4): 400 (1830). Briza capensis Thunb., Prodr. P1. Cap.: 21 (1794). Type: South Africa, Cape Province, Thunberg s.n. (holotype UPS). Fig. 1d.

Perennial, rhizomes short, base of culm with compacted dead sheaths. Culms 30-60 cm tall, erect or decumbent. Leaf-sheaths 7-10 cm long, margins and the lower surface pilose. Auricles pilose. Ligule a fringe of short hairs. Collars sparsely or densely pilose. Leaf-blades $10-25 \mathrm{~cm}$ by $2-3 \mathrm{~mm}$, both surfaces pilose. Panicles open, ovate to elliptic, $20-40$ by 3$5 \mathrm{~cm}$, branches glabrous. Spikelets $7-12$ by $2-4 \mathrm{~mm}$, with 8-14 fertile florets, ovate; florets tardily falling from the base of spikelet upwards, median groove prominent; rachilla persistent. L. glumes $1.8-2 \mathrm{~mm}$ 
long, tardily falling, lanceolate to elliptic, nerve with prominent ridge, keel scabrous, apex acute. Upper glumes $2-2.5 \mathrm{~mm}$ long, tardily falling, lanceolate to elliptic, 1-nerved with prominent ridge, keel scabrous, apex acute. Lemmas 3-4.2 mm long, caducous, ovate to elliptic, scabrous, chartaceous, apex acute. Paleas 3-3.5 mm long, tardily falling down, keel fimbriate, apex obtuse. Stamens 3; anthers 1.3-1.5 mm long. Caryopses $0.3-0.5 \mathrm{~mm}$ long, ellipsoid, terete, pericarp reticulate, dark brown.

Distribution.- Africa and Thailand.

Ecology.- In open pine forest and savannah (especially after fire).

Vernacular.- Yak on hep.

Notes. - This species is very similar in appearance to $E$. superba but differs by the absence of a wing on the palea keels and the spikelets do not disarticulate as a unit. It seems to be a rather rare species in Thailand since all collections originate from a single locality in pine forest near Mak Duk cliff at Phu Kradueng National Park, Loei Province.

Specimens examined.- Loei: Phu Kradueng National Park, 1300 m, 14, May 1951, T. Smitinand 415 (BKF); 18 March 1958, Th. Sørensen, K. Larsen \& B. Hansen 2244 (BKF, C, E); 26 March 2006, W. Chaisongkram 39 (KKU).

5. E. cilianensis (All.) Vignolo ex Janch., Mitt. Naturwiss. Vereins Univ. Wien 5: 110 (1907). P. cilianensis All., Fl. Pedem. 2: 246 (1785). Lectotype (designated by Vignolo 1904) ${ }^{13}$ : Italy, agro patrio Ciliana, C.A.L. Bellardi s.n. (TO; isotype picture K!). Fig. 1e.

Annual. Culms 40-70 cm long, erect or geniculately ascending. Leaf-sheaths 5-7 cm long with crateriform glands. Auricles bearded with long hairs. Ligules a fringe of hairs. Collars glabrous. Leafblades $5-20 \mathrm{~cm}$ by $2-10 \mathrm{~mm}$, margins and midrib glandular. Panicles open, ovate to elliptic, $20-30$ by $8-12 \mathrm{~cm}$, branches bearded in axils, sparse crateriform glands. Spikelets $3-15$ by $1.5-2 \mathrm{~mm}$, with $8-18$ fertile florets, oblong to ovate; florets falling from the base of spikelet upwards; rachilla persistent. L. glumes $1.8-2.3 \mathrm{~mm}$ long, caducous, ovate to elliptic, keel scabrous, apex acute. Upper glumes $2.2-2.5 \mathrm{~mm}$ long, caducous, ovate to elliptic, 3-nerved with lateral nerves reaching to margins, keel scabrous, apex acute. Lemmas 2.5-3 $\mathrm{mm}$ long, caducous, ovate, scaberulous, chartaceous, apex emarginate or acute. Paleas 2-2.5 mm long, persistent, keels ciliate, apex acute or bidentate. Stamens 3; anthers ca. $0.3 \mathrm{~mm}$ long. Caryopses $0.5-0.6 \mathrm{~mm}$ long, orbicular to subglobose, slightly laterally compressed, pericarp finely reticulate, reddish brown.

Distribution.- North and South America, Europe, Africa, Asia, Australasia, and Pacific.

Ecology.- In dry evergreen and deciduous forests.

Vernacular.- Ya khai hep yai.

Notes.- This species is easily recognized by having glands in most parts and its 3-nerved upper glume.

Specimens examined.- Chiang Mai: Doi Suthep National Park, 1100 m, 25 July 1958, Th. Sørensen, K. Larsen \& B. Hansen 4423 (BKF, C).

6. E. ciliaris (L.) R. Br., Congo: 478 (1818). P. ciliaris L. Syst. Nat. ed. 10, 2: 875 (1759). Lectotype (designated by Hitchcock 1908) ${ }^{14}$ : Jamaica, Herb. Linn. No. 87.66, Browne s.n. (LINN!).

Annual. Culms $5-10 \mathrm{~cm}$ tall, geniculately ascending. Leaf-sheaths $2-3 \mathrm{~cm}$ long, with tuberclebased hairs, margins ciliate. Auricles bearded with long hairs. Ligules a fringe of long hairs. Collars glabrous. Leaf-blades $3-5 \mathrm{~cm}$ by 1-2 $\mathrm{mm}$, both surfaces scabrous. Panicles spiciform to contracted, oblong, $12-14$ by $1.5-2 \mathrm{~cm}$, branches glabrous in axils. Spikelets $3-4$ by $1.5-2 \mathrm{~mm}$, with $6-10$ fertile florets, ovate to oblong; florets falling entire from the top of spikelet downwards; rachilla fragile. L. glumes $1.3-1.6 \mathrm{~mm}$ long, persistent, lanceolate to ovate, keel scabrous, margins ciliolate, apex acuminate. Upper glumes 1.8-2 mm long, persistent, ovate to elliptic, 1nerved, keel scabrous, apex acuminate. Lemmas 1.6$1.8 \mathrm{~mm}$ long, elliptic to ovate, scaberulous, membranous, apex obtuse to apiculate. Paleas $1.3-1.5 \mathrm{~mm}$ long, keel with tubercle-based hairs, apex obtuse. Stamens 2; anthers ca. $0.2 \mathrm{~mm}$ long. Caryopses $0.3-$ $0.4 \mathrm{~mm}$ long, ellipsoid, terete, pericarp finely striate, dark brown.

Distribution.- North and South America, Africa, Asia, Australasia, and Pacific.

Ecology.- Naturalised in Queen Sirikit Botanical Garden.

Vernacular.- Ya khai hao Muang.

Notes.- This is the first report of this species in Thailand. All specimens previously collected from Thailand were misidentified as E. ciliata which has a very similar appearance, but the latter has a hairy inflorescence axis.

Specimens examined.- Chiang Mai: Queen Sirikit Botanical Garden, 800 m, 24 May 1999, H. Hemadhulin 71 (QBG, E); East of nursery of Queen Sirikit Botanical Garden, 7 October 2001, S. Laegaard 21681 (AAU). 
7. E. ciliata (Roxb.) Nees, Fl. Bras. Enum. Pl. 2(1): 512 (1829). P. ciliata Roxb. Fl. Ind., ed. 1820, 1: 336 (1820). Type: India Orientalis, Heyne Benjamin 5015 (isotype BM!).-E. alopecuroides Balansa, J. Bot. (Morot) 4(9): 168 (1890). Type: Vietnam, Port de Cameran, 10 December 1886, Balansa s.n. (holotype $\mathrm{L}$ !, picture K!).

Perennial. Culms $15-70 \mathrm{~cm}$ tall, erect or geniculate. Leaf-sheaths 2-7 cm long, margins with tubercle-based hairs. Auricles bearded with long hairs. Ligules a fringe of long hairs. Collars hairy. Leafblades $10-15 \mathrm{~cm}$ by $1-2 \mathrm{~mm}$, both surfaces with tubercle-based hairs. Panicles spiciform, cylindrical or oblong, plumose, $2-8$ by $1-1.5 \mathrm{~cm}$, branches bearded in axils, axis hairy. Spikelets $5-7$ by $1-$ $1.5 \mathrm{~mm}$, with 7-8 fertile florets, ovate; florets falling entire from the top of spikelet downwards; rachilla fragile. L. glumes $1.5-2.3 \mathrm{~mm}$ long, persistent, lanceolate, 1-nerved, 1-keeled, keel scabrous, apex acuminate. Upper glumes $2-2.5 \mathrm{~mm}$ long, persistent, lanceolate, 1-nerved, 1-keeled, keel scabrous, apex acuminate. Lemmas $2.5-3 \mathrm{~mm}$ long, elliptic or oblong, scaberulous, membranous, apex apiculate. Paleas 1.3-2 mm long, keel with tubercle-based hairs, apex acute. Stamens 2; anthers ca. $0.25 \mathrm{~mm}$ long. Caryopses $0.4-0.5 \mathrm{~mm}$ long, orbicular, slightly laterally compressed, finely striate, dark brown.

Distribution.- Temperate and tropical Asia.

Ecology.-Common on sandy soil, sometimes on the beach.

Vernacular.- Ya khai hao.

Notes.- The distinctive characters of E. ciliata are its spiciform panicle and the inflorescence axis with long hairs.

Specimens examined.- Nakhon Ratchasima: 23 December 1923, A.F.G. Kerr 8086 (BK, BM, TCD). Chon Buri: A. Marcan 1371 (BM); Sattahip, 29 March 1971, J.F. Maxwell 71-202 (AAU, BK).

8. E. cumingii Steud., Syn. Pl. Glumac. 1: 266 (1854). Lectotype (designated by Lazarides, 1997): Philippines, Luzon, Cuming 1104 (K!; isotypes BM!, E!, G!, L!).- E. distans Hack., Publ. Bur. Sci. Gov. Lab. 35: 81 (1906). Type: Philippines, Kias, Luzon, June 1904, A.D.E. Elmer 6608 (holotype W, picture $\mathrm{K}$ !).

Annual. Culms 15-40 cm tall, erect or geniculate. Leaf-sheaths 5-10 cm long, glabrous. Auricles bearded with long hairs. Ligule a fringe of hairs. Collars glabrous. Leaf-blades $5-15 \mathrm{~cm}$ by $2-3 \mathrm{~mm}$, both surfaces with sparsely tubercle-based hairs. Panicles open, narrowly ovate to lanceolate, $15-30$ by 6-8 $\mathrm{cm}$, branches contracted or dense, bearded in axils. Spikelets $4-15$ by $1-1.5 \mathrm{~mm}$, with $14-20$ fertile florets, oblong; florets falling from the base of spikelet upwards, but rachilla fragile or ultimately breaking up from the top downwards; rachilla straight. L. glumes 1-1.3 mm long, caducous, lanceolate, keel scabrous, apex acuminate. Upper glumes 1-1.5 mm long, caducous, lanceolate to narrowly ovate, 1-nerved, keel scabrous, apex acuminate. Lemmas 1.6-2 mm long, caducous, elliptic to ovate, scaberulous to glabrous, membranous, apex acute to acuminate. Paleas 1$1.2 \mathrm{~mm}$ long, persistent, keel ciliate, apex acute. Stamens 3; anthers 0.1-0.2 mm long. Caryopses 0.3$0.5 \mathrm{~mm}$ long, ovoid or orbicular, slightly laterally compressed, pericarp finely reticulate, reddish brown.

Distribution.- America, Asia, and Australasia.

Ecology.- Common in open areas and on sandy soils.

Vernacular-_ Ya Philippines.

Notes.- This species is very similar in appearance to $E$. brownii. See also the note under $E$. brownii.

Specimens examined._Loei: Phu Ruea National Park, 22 November 2009, W. Chaisongkram 199 (KKU). Chanthaburi: Makham, $50 \mathrm{~m}, 13$ June 1963, K. Larsen 10058 (C). Ranong: Klong Naka, 4 February 1979, T. Koyama, C. Phengkhlai, P.J. O'Connor \& C. Niyomdham 15232 (AAU).

9. E. cylindrica (Roxb.) Nees ex Hook. \& Arn., Bot. Beechey Voy.: 251 (1838). P. cylindrica Roxb., Fl. Ind., ed. 1820, 1: 335 (1820). Type: China, Anonymous (holotype $\mathrm{K}$ !).

Perennial. Culms $20-40 \mathrm{~cm}$ tall, geniculately ascending. Leaf-sheaths $2-4 \mathrm{~cm}$ long, margins glabrous to densely hairy. Auricles bearded with long hairs. Ligules a fringe of hairs. Collars with a row of long hairs. Leaf-blades 3-10 $\mathrm{cm}$ by 4-7 $\mathrm{mm}$, both surfaces scabrous. Panicles spiciform panicles, cylindrical, $15-25$ by $1.5-2 \mathrm{~cm}$. Spikelets $5-6.5$ by $1-1.5 \mathrm{~mm}$, with 6-13 fertile florets, oblong; florets falling from the base of spikelet upwards; rachilla straight, persistent. L. glumes $1.2-1.4 \mathrm{~mm}$ long, caducous, ovate, keel scabrous, apex acute. Upper glumes 2-2.2 mm long, caducous, lanceolate to elliptic, 1-nerved, keel scabrous, apex acute or apiculate. Lemmas 2.2$2.5 \mathrm{~mm}$ long, caducous, ovate to elliptic, scaberulous, membranous, apex apiculate. Paleas 2-2.2 mm long, persistent, keels ciliolate to ciliate, slightly winged, apex obtuse. Stamens 3; anthers 0.4-0.5 mm long. Caryopses $0.3-0.4 \mathrm{~mm}$ long, sub-globose, pericarp striate to reticulate, brown.

Distribution.- China, Taiwan, Thailand and Japan.

Ecology.-Small tufts in open areas. 
Vernacular.- Ya krabok.

Notes.- This species is recognized by its spiciform panicle and the palea which has slightly winged keels.

Specimens examined.- Loei: Phu Kradueng National Park, 300 m, 22 March 1958, Th. Sørensen, $K$. Larsen \& B. Hansen 2419 (BKF, C). Nong Bua Lam Phu: Mueang, Ban Prao, 13 March 2010, W. Chaisongkram 193 (KKU).

10. E. diplachnoides Steud., Syn. Pl. Glumac. 1: 268 (1854). Type: Sudan, Kotschy 346 (holotype UPS; isotypes K!, L!).

Perennial, with short rhizomes. Culms 50$100 \mathrm{~cm}$ tall, erect or slightly geniculate. Leafsheaths $20-30 \mathrm{~cm}$ long, margins glabrous. Auricles glabrous. Ligules a fringe of hairs. Collars glabrous. Leaf-blades $10-25 \mathrm{~cm}$ by $3-5 \mathrm{~mm}$, both surfaces scabrous. Panicles open, lanceolate, $30-50$ by 12 $16 \mathrm{~cm}$, branches sub-digitate with the lowermost branch longest, the lowermost branch 6-8 cm long. Spikelets $2-3$ by $1-1.5 \mathrm{~mm}$, with $4-6$ fertile florets, elliptic; florets falling entire from the top of spikelet downwards; rachilla fragile. L. glumes $0.4-0.5 \mathrm{~mm}$ long, persistent, ovate, keel glabrous to scaberulous, apex acute. Upper glumes $0.6-0.7 \mathrm{~mm}$ long, persistent, broadly ovate, 1-nerved, keel glabrous, apex acute. Lemmas $0.8-1 \mathrm{~mm}$ long, broadly ovate, glabrous, membranous, apex acute. Paleas 0.8 $0.9 \mathrm{~mm}$ long, keels ciliate, apex acute. Stamens 2; anthers ca. $0.2 \mathrm{~mm}$ long. Caryopses $0.4-0.5 \mathrm{~mm}$ long, ellipsoid, terete, pericarp smooth to finely striate and reticulate, brown.

Distribution.- Tropical and subtropical Africa and Asia.

Ecology.- Common in swampy areas or paddy fields.

Vernacular.— Ya khai pu.

Notes.- This species is distinctive sub-digitate branches of which the lowest one is the longest. It is easily confused with E. japonica, a species with an oblong panicle.

Specimens examined.- Nakhon Sawan: south of Nakhon Sawan, $100 \mathrm{~m}, 15$ March 1958, Th. Sørensen, K. Larsen \& B. Hansen 1939 (BKF, C, E). Khon Kaen: 29 October 2001, S. Laegaard \& M. Norsaengsri 21868 (AAU, QBG, K). Nakhon Ratchasima: 20 December 1923, A.F.G. Kerr 8083 (BM, K, TCD). Kanchanaburi: 12 July 1930, A.F.G. Kerr 19542 (BK, BM). Songkhla: Khao Rak Kiat, 25 m, 9 December 1986, J.F. Maxwell 86-1043 (AAU, $\mathrm{BKF})$.
11. E. gangetica (Roxb.) Steud., Syn. Pl. Glumac. 1: 266 (1854). P. gangetica Roxb., Fl. Ind. ed. 1820, 1: 341 (1820). Type: India, Roxburgh s.n. (holotype $\mathrm{K}$; isotype BM!).—E. stenophylla Hochst. ex Miq., Anal. Bot. Ind. 2: 27 (1851). Type: India, prope urbem Mangalor, Metz 664 (holotype L; isotype G!). Fig. 1f.

Annual. Culms $40-80 \mathrm{~cm}$ tall, geniculately ascending. Leaf-sheaths $5-10 \mathrm{~cm}$ long, glabrous. Auricles bearded with hairs. Ligules ciliolate membranous. Collars glabrous. Leaf-blades $10-25 \mathrm{~cm}$ by $5-8 \mathrm{~mm}$, upper surface sparsely hairy. Panicles open, ovate, $15-30$ by $6-8 \mathrm{~cm}$. Spikelets $6-12$ by 1-1.5 mm, with 15-26 fertile florets, oblong; florets falling from the base of spikelet upwards, often pale green; rachilla persistent. L. glumes 1-1.2 mm long, caducous, lanceolate, keel scaberulous, apex acute. Upper glumes 1.5-1.8 mm long, caducous, elliptic to ovate, 1-nerved, keel scaberulous to scabrous, apex acute. Lemmas 1.3-1.8 mm long, caducous, elliptic, glabrous, membranous, apex acute. Paleas 1$1.2 \mathrm{~mm}$ long, tardily falling, keels scabrous, apex acute to obtuse. Stamens 2; anthers $0.2-0.3 \mathrm{~mm}$ long. Caryopses $0.4-0.6 \mathrm{~mm}$ long, orbicular, slightly laterally compressed, pericarp striate and reticulate, dark brown.

Distribution.- North and South America, Africa and Tropical Asia.

Ecology.- On sandy soils and at roadsides.

Vernacular.- Ya krok lek.

Notes.- The appearance of this species is similar to $E$. atrovirens but it is normally smaller and its palea ultimately disarticulates. It was treated by $\operatorname{Stapf}^{15}$ of $E$. stenophylla which matches the type of $P$. gangetica housed in BM, but the name E. gangetica has priority. Veldkamp ${ }^{10}$ noted that $E$. gangetica is very similar to $E$. nutans, a perennial species with three stamens.

Specimens examined.- Chiang Mai: Doi Suthep National Park, 1100 m, 12 July 1912, A.F.G. Kerr 2623 (BM, K, TCD). Loei: $16^{\circ} 52^{\prime} \mathrm{N} 101^{\circ} 52^{\prime}$ E, 1300 m, 23 December 1971, C.F. van Beusekom, $R$. Geesink, C. Phengkhlai \& B. Wongwan 4511 (BKF, C). Ubon Ratchathani: Sirindhon, Khun Rai, 7 October 2009, W. Chaisongkram 180 (KKU). Surat Thani: 1 August 1927, A.F.G. Kerr 13060 (BM).

12. E. japonica (Thunb.) Trin., Mém. Acad. Imp. Sci. St.-Pétersbourg, Sér. 6, Sci. Math. 1(4): 405 (1830). P. japonica Thunb., Fl. Jap.: 51 (1784). Type: Japan, Herb. Thunberg 2252 (holotype UPS, photo K!).- E. aurea Steud., Syn. Pl. Glumac. 1: 267 (1854). Type: Philippines, Laguna, Cuming 545 (holotype P; isotypes BM!, G!-3 sheets, L). Fig. 1g. 
Annual. Culms 40-70 cm tall, erect. Leaf-sheaths $10-30 \mathrm{~cm}$ long, margins glabrous. Auricles glabrous. Ligules frimbriate membranous. Collars glabrous. Leaf-blades $10-20 \mathrm{~cm}$ by $2-4 \mathrm{~mm}$, both surfaces scabrous. Panicles contracted to open, oblong, 30-50 by $4-5 \mathrm{~cm}$, branches whorled, the lowermost branch $2-2.5 \mathrm{~cm}$ long, axis scabrous. Spikelets $1-3$ by 0.8 $1 \mathrm{~mm}$, with 4-6 fertile florets, ovate; florets falling entire from the top of spikelet downwards; rachilla fragile. L. glumes $0.6-0.8 \mathrm{~mm}$ long, persistent, ovate to lanceolate, keel scabrous to scaberulous, apex acute. Upper glumes $0.8-1 \mathrm{~mm}$ long, persistent, broadly ovate, 1-nerved, keel scaberulous, apex acute. Lemmas $0.5-0.7 \mathrm{~mm}$ long, broadly ovate, scaberulous, membranous, apex obtuse. Paleas $0.4-0.6 \mathrm{~mm}$ long, keels ciliate, apex obtuse. Stamens 2; anthers ca. $0.2 \mathrm{~mm}$ long. Caryopses $0.2-0.3 \mathrm{~mm}$ long, ellipsoid, terete, pericarp rugulose, dark brown.

Distribution.- America, Europe, Africa, and Asia.

Ecology.- Tufted, common in paddy fields and on muddy ground.

Vernacular.- Ya khai pu lek.

Notes.- This species is distinct from other species by virtue of its whorled or sub-digitate panicles and oblong inflorescences.

Specimens examined.- Chiang Mai: Doi Inthanon National Park, 500 m, 19 January 1969, H.P. Nooteboom, B. Tantisewie \& C. Phengkhlai 767 (BKF). Uttaradit: Den Chai, 27 January 2007, W. Chaisongkram 106 (KKU). Sakon Nakhon: Phu Phan National Park, 16 November 1962, P. Suvarnakoses 1901 (BKF, C, E). Kanchanaburi: Sai Yok, 170 m, 29 December 1961, K. Larsen 9035 (C). Sa Kaeo: 16 October 1928, Put 1975 (BK, BM).

13. E. montana Balansa, J. Bot. (Morot) 4(8): 168 (1890). Type: Cambodia, Pusat Mt., F.F. Godefroy 487 (holotype L!).- E. malayana Stapf, Fl. Brit. India 7(22): 317 (1896). Lectotype (designated by Veldkamp 2002): India, Perak, Wray 773 (K!). Fig. $1 \mathrm{~h}$.

Annual. Culms $20-50 \mathrm{~cm}$ tall, erect to decumbent. Leaf-sheaths 2-8 cm long, glabrous. Auricles bearded with long hairs. Ligules a fringe of long hairs. Collars glabrous. Leaf-blades $3-15 \mathrm{~cm}$ by $1-3 \mathrm{~mm}$, upper surface sparsely hairy, lower surface glabrous. Panicles open to contracted, ovate to oblong, 5-25 by $1.5-5 \mathrm{~cm}$, bearded in axils. Spikelets $3-10$ by $1.5-$ $2 \mathrm{~mm}$, with 7-30 fertile florets, oblong to elliptic; florets falling from the base of spikelet upwards; rachilla straight, persistent. L. glumes $0.7-1 \mathrm{~mm}$ long, caducous, ovate, keel scabrous, apex acute. Upper glumes $0.8-1.8 \mathrm{~mm}$ long, caducous, broadly ovate, 1 nerved, keel scabrous, apex acute. Lemmas 1-2 mm long, caducous, broadly ovate, glabrous to scaberulous, membranous, apex acute. Paleas 1-1.4 mm long, persistent, glabrous, apex acute. Stamens 3; anthers ca. $0.3 \mathrm{~mm}$ long. Caryopses $0.4-0.5 \mathrm{~mm}$ long, ellipsoid, terete, pericarp smooth to finely reticulate, reddish brown.

Distribution.- Temperate and Tropical Asia.

Ecology.-Common in dry deciduous forest.

Vernacular.- Ya langka.

Notes.-E. montana is similar to E. brownii but differs by its continuous and persistent rachilla.

Specimens examined.- Chiang Mai: Doi Inthanon National Park, 800 m, 12 December 1969, C.F. van Beusekom \& C. Phengkhlai 2488 (AAU, C). Loei: Phu Kradueng National Park, 27 March 2006, W. Chaisongkram 52 (KKU). Nakhon Ratchasima: 28 July 2008, M. Norsaengsri 3987 (QBG). Ubon

Ratchathani: Soi Sawan waterfall, 10 July 2009, W. Chaisongkram 178 (KKU). Kanchanaburi: 31 December 1924, A.F.G. Kerr 9803 (BM). Phetchaburi: Cha-am, 8 April 1924, A. Marcan 1686 (BM); Thung Luang, 10 November 1931, A.F.G. Kerr 20628 (BM). Prachuap Khiri Khan: 6 November 1927, A.F.G. Kerr 13455 (BM); 25 m, 12 August 1966, K. Larsen, T. Smitinand \& E. Warncke 1516 (AAU). Saraburi: Sam Lahn, 100 m, 16 June 1974, J.F. Maxwell 74-606 (AAU, L). Chanthaburi: Makham, 15 m, 8 November 2008, W. Chaisongkram 155 (KKU); 16 February 2009, M. Norsaengsri 4915 (QBG). Narathiwat: Tak Bai, near sea level, 6 February 1988, C. Niyomdham 1655 (AAU, C, E, L).

14. E. nigra Nees ex Steud., Syn. Pl. Glumac. 1: 267 (1854). Lectotype (designated by Veldkamp 2002): Peninsular India Orientalis, Herb. Wight proper. 1782 (LE; isotypes G!, K!).- E. atropurpurea Hochst. ex Steud., Syn. Pl. Glumac. 1: 267 (1854). Type: India, Tamil Nadu, Coimbatore, Nilgiri, R.F. Hohenacker 938 (isotypes BM!, E!, G!-2 sheets).

Perennial. Culms $15-40 \mathrm{~cm}$ tall, erect, glabrous. Leaf-sheaths $2-4 \mathrm{~cm}$ long, margins and adjacent zones with tubercle-based hairs. Auricles bearded with long hairs. Ligule a fringe of hairs. Collars with a row of long hairs. Leaf-blades $5-15 \mathrm{~cm}$ by $3-6 \mathrm{~mm}$, upper surface sparsely hairy, lower surface scabrous. Panicles open, $20-30$ by $6-8 \mathrm{~cm}$, peduncle and axis glabrous, branches glabrous. Spikelets $4-6$ by $1.5-$ $2 \mathrm{~mm}$, with 4-6 (-7) fertile florets, ovate, dark purple; florets falling from the base of spikelet upwards; rachilla zig-zag, persistent. L. glumes $1.2-1.5 \mathrm{~mm}$ long, caducous, lanceolate, keel glabrous, apex acumi- 
nate. Upper glumes 1.8-2.2 mm long, caducous, lanceolate, 1-nerved, keel scabrous, apex acuminate. Lemmas 1.6-2.0 mm long, caducous, ovate to elliptic, scaberulous, membranous and hyaline near the apex, apex obtuse to acute. Paleas $1.5-1.7 \mathrm{~mm}$ long, persistent, keels glabrous, apex bidentate. Stamens 3; anthers ca. $0.6 \mathrm{~mm}$ long. Caryopses $0.5-0.6 \mathrm{~mm}$ long, trigonous to reniform, pericarp striate and reticulate, dark brown.

Distribution.- Tropical and Temperate Asia.

Ecology.-Common along roadsides.

Vernacular.- Ya khai mang da.

Notes.- This species is recognized by its dark purple and strongly imbricate spikelets.

Specimens examined.-Chiang Mai: Doi Khun Huai Pong, 1800 m, 5 March 1968, B. Hansen \& T. Smitinand 12831 (AAU, BKF, C, E, L); at the summit of Doi Inthanon National Park, 3 September 2008, W. Chaisongkram 141 (KKU).

15. E. nutans (Retz.) Nees ex Steud., Nomencl. Bot., ed. 2, 1: 563 (1840). P. nutans Retz., Observ. Bot. 4: 19 (1786). Lectotype (designated by Bor 1959) ${ }^{16}$ : India, Tranquebaria, Koenig s.n. (LD, isotypes $\mathrm{C} !-2$ sheets,).

Perennial. Culms $25-40 \mathrm{~cm}$ tall, geniculately ascending. Leaf-sheaths $2-5 \mathrm{~cm}$ long, margins glabrous. Auricles glabrous to sparsely hairy. Ligules a fringe of hairs. Collars glabrous. Leaf-blades $7-12 \mathrm{~cm}$ by 5-10 mm, lanceolate to oblong, upper surface hairy, lower surface scaberulous. Panicles contracted, oblong to narrowly elliptic, $15-20$ by $3-4 \mathrm{~cm}$. Spikelets $5-20$ by $2-2.5 \mathrm{~mm}$, with $6-25$ fertile florets, oblong; florets falling from the base of spikelet upwards; rachilla straight, persistent. L. glumes $1.5-1.8 \mathrm{~mm}$ long, caducous, lanceolate, keel scabrous, apex acute. Upper glumes 1.8-2 mm long, caducous, elliptic, 1nerved, keel scabrous, apex acute. Lemmas 2-2.2 mm long, caducous, elliptic, scaberulous, membranous, apex acute to acuminate. Paleas $1.5-1.8 \mathrm{~mm}$ long, persistent, keels ciliolate, apex bidentate. Stamens 3; anthers $0.5-0.6 \mathrm{~mm}$ long. Caryopses $0.4-0.5 \mathrm{~mm}$ long, orbicular, slightly laterally compressed, reticulate, brown.

Distribution.- North America, Africa, and Asia.

Ecology.- In open areas and moist locations.

Vernacular.- Ya khaem.

Notes. - This species is recognized by having very long oblong spikelets and contracted panicles.

Specimens examined.- Kanchanaburi: 1030 m, Thong Pha Phum, 25 January 2006, W. Chaisongkram 83 (KKU). Chanthaburi: Makham, 30 March 2006, W. Chaisongkram 53 (KKU); 16
February 2009, M. Norsaengsri 4907 (QBG).

16. E. pilosa (L.) P. Beauv., Ess. Agrostogr.: 162 (1812). P. pilosa L., Sp. Pl. 1: 68 (1753). Epitype (designated by Scholz 2000) ${ }^{17}$ : Italy, an Wegen zwischen den Reisfeldern von Oldenico unweit Vercelli in Oberitaliën, Kneucker 344 (B; isoepitype L!).

Annual. Culms 8-30 cm tall, erect or geniculate. Leaf-sheaths $1-3 \mathrm{~cm}$ long, margins glabrous. Auricles bearded with long hairs. Ligules a fringe of hairs. Collars glabrous. Leaf-blades $2-15 \mathrm{~cm}$ by $2-3 \mathrm{~mm}$, upper surface scabrous, lower surface striate. Panicles open to contracted, lanceolate to ovate, $3-25$ by $5-$ $7 \mathrm{~cm}$, branches flexuous, bearded in axils or glabrous, sub-digitate at lower nodes. Spikelets $3-4$ by $0.5-$ $0.8 \mathrm{~mm}$, with 3-9 fertile florets, ovate to narrowly lanceolate; florets falling from the base of spikelet upwards; rachilla zig-zag, persistent. L. glumes 0.5$0.8 \mathrm{~mm}$ long, caducous, narrowly lanceolate, keel obscure, apex apiculate. Upper glumes $1-1.5 \mathrm{~mm}$ long, caducous, lanceolate, 1-nerved, keel scaberulous, apex acuminate. Lemmas 1.2-1.5 mm long, caducous, ovate to elliptic, scaberulous, membranous, apex acute. Paleas tardily falling, $1.1-1.3 \mathrm{~mm}$ long, keels scaberulous. Stamens 3; anthers ca. $0.1 \mathrm{~mm}$ long. Caryopses $0.3-0.5 \mathrm{~mm}$ long, reniform, terete, pericarp smooth to finely reticulate, dark brown.

Distribution.- America, Europe, Africa, Asia, Australia, and Pacific.

Ecology.-Common along roadsides.

Vernacular.- Ya ko.

Notes.- This species has narrow spikelets and it is very similar to E. tenuifolia in the shape of its spikelets (but its spikelets are smaller).

Specimens examined.- Chiang Mai: Chom Thong, Ban Huai Nam Kow, Yahng Krahm subdistrict, 350 m, 3 October 1991, J.F. Maxwell 91-838 (E, L). Nong Bua Lam Phu: Mueang, Ban Prao, $W$. Chaisongkram 93 (KKU). Chanthaburi: Makham, 8 November 2008, W. Chaisongkram 151 (KKU). Nakhon Si Thammarat: Ko Chum Thung, 50 m, 30 January 1928, A.F.G. Kerr 14349 (BM, K, TCD).

17. E. riparia (Willd.) P. Beauv., Ess. Agrostogr.: 162 (1812). P. riparia Willd., Ges. Naturf. Freunde Berlin Neue Schriften 4: 185 (1803). Type: India, Madras, Rottler s.n. in Herb. Willdenow 1940/2 (holotype $\mathrm{B}$, fragm. $\mathrm{K}$ !).

Annual or perennial. Culms $20-30 \mathrm{~cm}$ tall, geniculately ascending. Leaf-sheaths $2-4 \mathrm{~cm}$ long, margins glabrous sometimes ciliate or sparsely pilose in the uppermost part. Auricles bearded with long hairs. Ligules a fringe of hairs. Collars pilose. Leaf-blades 3-8 cm long, both surfaces scaberulous. Panicles open 
with compact branches, ovate to lanceolate, $4-8$ by 2-2.5 cm, branches bearded in axils. Spikelets 2-4 by $1-1.5 \mathrm{~mm}$, with $5-10$ fertile florets, lanceolate to oblong; florets falling entire from the top of spikelet downwards; rachilla fragile. L. glumes $0.8-1.5 \mathrm{~mm}$ long, ultimately falling, ovate to lanceolate, keel scabrous, apex acute. Upper glumes $1.2-1.5 \mathrm{~mm}$ long, ultimately falling, lanceolate to ovate, 1-nerved, keel scabrous, apex acute. Lemmas $1.3-1.5 \mathrm{~mm}$ long, ovate to elliptic, scaberulous, membranous, apex obtuse to acute. Paleas 1.2-1.5 mm long, keel with tuberclebased hairs, apex obtuse. Stamens 3; anthers ca. $0.4 \mathrm{~mm}$ long. Caryopses $0.3-0.4 \mathrm{~mm}$ long, ellipsoid, terete, pericarp rugulose, dark brown.

Distribution.- Tropical Asia.

Ecology.- Common in open areas on a range of soil types.

Vernacular.- Ya khai hao.

Notes.- E. riparia is very similar to E. tenella but differs by having open panicle and compact branches.

Specimens examined.- Sakon Nakhon: $25 \mathrm{Au}-$ gust 2007, W. Chaisongkram 114 (KKU). Prachuap Khiri Khan: 7 August 1966, K. Larsen, T. Smitinand \& E. Warncke 1199 (TCD); K. Larsen, T. Smitinand \& E. Warncke 1247 (AAU, K).

18. E. superba Peyr., Sitzungsber. Kaiserl. Akad. Wiss., Math.-Naturwiss. Cl. 38: 584 (1860). Type: Angola, Benguela, Wawra 244 (holotype W). Fig. 1 i.

Perennial, Culms $40-60 \mathrm{~cm}$ tall, geniculate. Leafsheaths 5-8 cm long, loose, margins glabrous. Auricles bearded with long hairs. Ligules a fringe of long hairs. Collars glabrous. Leaf-blades $10-30 \mathrm{~cm}$ by 4-8 $\mathrm{mm}$, upper surface hairy, lower surface glabrous. Panicles loose, irregular, $10-20$ by $2-3 \mathrm{~cm}$, branches glabrous or bearded in axils. Spikelets $7-12$ by $3-$ $4(-5) \mathrm{mm}$, with 4-12 fertile florets, broadly elliptic to ovate, breaking up below the glumes and fallen down as a whole. L. glumes $2.5-3 \mathrm{~mm}$ long, persistent, ovate, keel scabrous, apex obtuse. Upper glumes 3$4 \mathrm{~mm}$ long, persistent, ovate, 1-nerved, keel scabrous, apex obtuse. Lemmas 3-5 mm long, lanceolate to ovate, scaberulous, membranous, apex obtuse. Paleas 2-4.5 mm long, keel winged, ciliolate on wings, margins glabrous, apex obtuse. Stamens 3; anthers 1.6$1.8 \mathrm{~mm}$ long. Caryopses $0.5-0.7 \mathrm{~mm}$ long, reniform, terete, pericarp reticulate, brown.

Distribution.- North and South America, Africa, Asia, Australasia, and Pacific.

Ecology.- Introduced from India for fodder as a pasture species.

Vernacular.- Ya yung ram phan.
Notes. - The species can be easily recognized by its rather large spikelets that are up to $5 \mathrm{~mm}$ wide and its strongly imbricate florets. Moreover, the wings on palea keels are prominently seen in lateral view.

Specimens examined.- Nakhon Ratchasima: Pak Chong, April 1969, S.N. (BKF).

19. E. tef (Zucc.) Trotter, Boll. Soc. Bot. Ital.: 62 (1918). P. tef Zucc. Diss. Ditef. (1775). Type: Cultivated in Florence from seed collected in Ethiopia, Anonymous (holotype FI).

Perennial, rhizomes short. Culms $20-60 \mathrm{~cm}$ tall, erect or geniculate. Leaf-sheaths $3-10 \mathrm{~cm}$ long, margins glabrous. Auricles bearded with long hairs. Ligules a fringe of hairs. Collars glabrous. Leaf-blades $10-20 \mathrm{~cm}$ by $2-4 \mathrm{~mm}$, both surfaces scabrous. Panicles open, lanceolate to ovate, 2540 by $3-6 \mathrm{~cm}$, branches flexuous, bearded in axils or glabrous, whorled at lower nodes. Spikelets 58 by $1-1.2 \mathrm{~mm}$, with $4-6$ fertile florets, lanceolate, green; florets tardily falling from the base of spikelet upwards; rachilla zig-zag, persistent. L. glumes 1.5$1.8 \mathrm{~mm}$ long, tardily falling, lanceolate, keel scabrous, apex acuminate. Upper glumes $2-2.5 \mathrm{~mm}$ long, tardily falling, lanceolate, 1-nerved, keel scabrous, apex acuminate. Lemmas $2.2-2.7 \mathrm{~mm}$ long, tardily falling, elliptic to lanceolate, scabrous, slightly membranous to scarious, apex acute. Paleas $1.8-2 \mathrm{~mm}$ long, persistent, keels scaberulous, apex acute. Stamens 3; anthers 0.3-0.4 mm long. Caryopses $0.9-1 \mathrm{~mm}$ long, reniform, terete, pericarp finely striate and reticulate, dark brown.

Distribution.- North and South America, Europe, Africa, Asia, Australasia, and Pacific.

Ecology.-Cultivated species.

Vernacular.- Ya teff.

Notes.-E. tef is a staple cereal in Africa. It was introduced to Thailand in 1929. The label note from the only specimen collected from Thailand indicates that it was "introduced two years ago".

Specimens examined.- Nakhon Ratchasima: Pak Chong, 1-200 m, 18 May 1931, A.F.G. Kerr s.n. (BM).

20. E. tenella (L.) P. Beauv. ex Roem. et Schult. Syst. Veg. 2: 576 (1817). P. tenella L. Sp. Pl. 1: 69 (1753). Lectotype (designated by Mitra \& Jain 1980) ${ }^{18}$ : India, Herb. Linn. No. 87.33, Anonymous (LINN!).- E. amabilis (L.) Wight \& Arn. ex Nees, Bot. Beechey Voy.: 251 (1838). P. amabilis L. Sp. Pl. 1: 68 (1753). Lectotype (designated by Veldkamp 2000): Sri Lanka, Herb. Hermann 2: 59, no. 46, Anonymous (BM!). Fig. 1j. 
Small annual. Culms $10-30 \mathrm{~cm}$ tall, erect or geniculate, slightly compressed or terete. Leafsheaths $5-15 \mathrm{~cm}$ long, glabrous or hairy near the blade, margins with tubercle-based hairs. Auricles bearded with long hairs. Ligules a fringe of long hairs. Collars with a row of long hairs. Leaf-blades $7-15 \mathrm{~cm}$ by $8-12 \mathrm{~mm}$, upper surface scabrous, lower surface glabrous. Panicles open, lanceolate to ovate, 7-20 by $3-8 \mathrm{~cm}$, axis scabrous, branches with long hairs in axils, branchlet and pedicel glandular. Spikelets $1.5-2$ by $0.8-1 \mathrm{~mm}$, with $4-7$ fertile florets, delicate, ovate to ovate-oblong; florets falling entire from the top of spikelet downwards; rachilla fragile. L. glumes $0.4-0.5 \mathrm{~mm}$ long, persistent, narrowly lanceolate, keel scaberulous, apex acute. Upper glumes $0.5-0.7 \mathrm{~mm}$ long, persistent, elliptic, 1-nerved, keel scaberulous, apex acute. Lemmas $1.5-2 \mathrm{~mm}$ long, broadly ovate or oblong, scaberulous, membranous, apex mucronulate, acute or muticous. Paleas $0.8-0.9 \mathrm{~mm}$ long, keels with tubercle-based hairs, apex obtuse. Stamens 3; anthers ca. $0.2 \mathrm{~mm}$ long. Caryopses $0.3-0.4 \mathrm{~mm}$ long, ellipsoid, terete, pericarp finely rugulose, brown.

Distribution.- North and South America, Africa, Asia, Australasia, and Pacific.

Ecology.- Widespread and common in lowlands in elevations from sea-level to $2000 \mathrm{~m}$, often in shady sites, grasslands and dry forests.

Vernacular.- Ya khai hep lek.

Notes.- E. tenella is one of the commonest grasses in Thailand and can be recognized by its delicate spikelets that are often pinkish, and by the presence of tubercle-based hairs on its palea keels. Following Jansen ${ }^{19}$, who discussed the names E. amabilis and E. tenella, we have accepted the name E. tenella since it was chosen by $\operatorname{Stapf}^{15}$ who first united.

Specimens examined.- Chiang Mai: Tham Chiang Dao, 350 m, 17 February 1958, Th. Sørensen, K. Larsen \& B. Hansen 1211 (C); 1221 (BKF, C, E). Nong Bua Lam Phu: Ban Prao, 15 September 2006, W. Chaisongkram 92 (KKU). Prachuap Khiri Khan: Khao Chong Krachock, 18 August 1967, T. Shimizu, N. Pukuoka \& A. Nalamphun T7610 (BKF); 25 December 2007, W. Chaisongkram 125 (KKU). Krung Thep Maha Nakhon: Paved path, 15 October 1922, A. Marcan 1022 (BM). Narathiwat: Tak Bai, near sea level, 18 September 1987, C. Niyomdham \& D. Sriboonma 1628 (AAU, BKF, E).

21. E. tenuifolia (A. Rich.) Hochst. ex Steud., Syn. Pl. Glumac. 1: 268 (1854). P. tenuifolia A. Rich., Tent. Fl. Abyss. 2: 425 (1851). Lectotype (designated by Phillips 1995) ${ }^{20}$ : Ethiopia, in locis incultis Vallium prope Adoam, Schimper 92 (P; isotypes K!, G!, L).E. parviglumis Hochst. ex Steud., Syn. Pl. Glumac. 1: 267 (1854). Type: India, Tamil Nadu, Coimbatore, in montibus Nilagiri, Metz 936 (isotypes G!-2 sheets). Fig. 1k.

Perennial. Culms $30-70 \mathrm{~cm}$ tall, erect or geniculate, slightly compressed, lateral branches lacking. Leaf-sheaths 5-15 cm long, coriaceous to membranous, margins with tubercle-based hairs. Auricles bearded with long hairs. Ligules a fringe of long hairs. Collars glabrous. Leaf-blades $10-25 \mathrm{~cm}$ by $2-3 \mathrm{~mm}$, upper surface scabrous, lower surface glabrous. Panicles open, lanceolate to ovate, 20-40 by $8-10 \mathrm{~cm}$, branches bearded in axils. Spikelets $6-$ 10 by $1-1.5 \mathrm{~mm}$, with $8-15$ fertile florets lanceolate; florets falling from the base of spikelet upwards; rachilla zig-zag, persistent. L. glumes $0.5-0.8 \mathrm{~mm}$ long, caducous sometimes tardily falling, narrowly ovate, keel scaberulous, apex acute. Upper glumes 1$2 \mathrm{~mm}$ long, caducous sometimes tardily falling, ovate to lanceolate, 1-nerved, keel scabrous, apex acute. Lemmas 2-2.5 mm long, caducous, ovate to oblong, scaberulous, membranous, apex acute. Paleas 1. 8$2 \mathrm{~mm}$ long, persistent, keel ciliolate, apex bidentate. Stamens 2-3; anthers ca. $0.5 \mathrm{~mm}$ long. Caryopses $0.5-0.6 \mathrm{~mm}$ long, trigonous, pericarp finely reticulate, reddish brown, adhered to palea.

Distribution.- North and South America, Europe, Africa, Asia, Australasia, and Pacific.

Ecology.- In open areas at 600-2560 m.

Vernacular.- Ya khamao.

Notes.- This species commonly exhibits dark green spikelets and is similar to E. nigra but differs by having more narrowly lanceolate spikelets and caryopses that adhere to the palea after lemma shedding.

Specimens examined.- Chiang Mai: Doi Suthep National Park, 1575 m, 22 May 1988, J.F. Maxwell 88-670 (AAU, BKF, L); Doi Inthanon National Park, 3 September 2008, W. Chaisongkram 130 (KKU). Tak: Um Phang, 14 November 2008, W. Chaisongkram 161 (KKU). Loei: Phu Ruea National Park, 21 November 2009, W. Chaisongkram 203 (KKU).

22. E. unioloides (Retz.) Nees ex Steud., Syn. Pl. Glumac. 1: 264 (1854). P. unioloides Retz., Observ. Bot. 5: 19 (1788). Type: India, 1776, Koenig s.n. (holotype LD, picture K!, fragment K!; isotypes BM!, K). Fig. 11.

Annual or perennial, sometimes rhizomatous. Culms $10-50 \mathrm{~cm}$ tall, erect or geniculate, internodes glabrous, sometimes rooted at lower nodes. Leafsheaths $2-5 \mathrm{~cm}$ long, margins hyaline, glabrous. Au- 
ricles bearded with long hairs. Ligules a fringe of hairs. Collars glabrous. Leaf-blades $5-20 \mathrm{~cm}$ by 1-8 $\mathrm{mm}$, upper surface sparsely hairy, lower surface striate. Panicles open, ovate, obovate to elliptic, 535 by $6-8 \mathrm{~cm}$, branches glabrous. Spikelets $5-12$ by 2.5-4 (-5) mm, normally pinkish, with 3-30 fertile florets, ovate; florets falling from the base of spikelet upwards; rachilla persistent. L. glumes $1.8-2.3 \mathrm{~mm}$ long, caducous, narrowly lanceolate, keel scaberulous, apex acuminate. Upper glumes 2-2.4 mm long, caducous, lanceolate, 1-nerved, keel scaberulous, apex acuminate. Lemmas 1.5-2.5 mm long, caducous, ovate, scaberulous to scabrous, membranous, apex acute. Paleas 1.5-2.2 mm long, caducous, keels ciliolate, apex bidentate. Stamens 2-3; anthers ca. $0.3 \mathrm{~mm}$ long. Caryopses $0.5-0.6 \mathrm{~mm}$ long, orbicular, laterally compressed, pericarp reticulate, dark brown.

Distribution.- - North and South America, Africa, Asia, and Australasia.

Ecology.- Common on sandy soil and in open area in dipterocarp forest, often found on wet soils.

Vernacular.- Ya krok.

Notes. - This species is recognized by its broadly elliptic or slightly ovate spikelets. The spikelet width is up to $5 \mathrm{~mm}$ and they are commonly pinkish.

Specimens examined.- Mae Hong Son: $500 \mathrm{~m}$, 9 September 1974, K. Larsen \& S.S. Larsen 34315 (AAU, BKF, C, E). Chiang Mai: Pha Mon, Doi Inthanon National Park, 4 September 2008, W. Chaisongkram 140 (KKU). Loei: Phu Kradueng National Park, 1300 m, 29 November 1958, Th. Sorensen, K. Larsen \& B. Hansen 6318 (C, K). Kanchanaburi: Sai Yok, 25 November 1971, C.F. van Beusekom, R. Geesink, C. Phengkhlai \& B. Wongwan 3948 (BKF, C). Nakhon Nayok: Khao Yai National Park, 350 m, 20 November 2002, J.F. Maxwell 02-433 (BKF). Prachin Buri: Krabin, 8 November 1930, A. Marcan 2530 (BM, L); 10 November 1930, A. Marcan 2592 (BM, K). Narathiwat: 6 February 1988, C. Niyomdham 1655 (AAU, BKF, C, E, L).

23. E. viscosa (Retz.) Trin., Mém. Acad. Imp. Sci. St.-Pétersbourg, Sér. 6, Sci. Math. 1(4): 397 (1830). P. viscosa Retz., Observ. Bot.: 20 (1786). Type: India, Koenig in Herb. Retzius s.n. (holotype LD, fragment $\mathrm{K}$ !; isotype $\mathrm{BM}$ !).

Annual. Culms $20-45 \mathrm{~cm}$ tall, erect or geniculate, with sticky glandular patches below the nodes. Leafsheaths 3-6 cm long, glabrous or sparsely hairy. Auricles glabrous. Ligule a fringe of hairs. Collars hairy. Leaf-blades $6-20 \mathrm{~cm}$ by $3-8 \mathrm{~mm}$, both surfaces glabrous. Panicles open, oblong to ovate, $15-25$ by $5-8 \mathrm{~cm}$, glabrous or pilose in axils, branchlet and pedicel with pale brown glands. Spikelets 2.5-4.5 by $0.8-1 \mathrm{~mm}$, with $3-10$ fertile florets, oblong to ovate; florets falling entire from the top of spikelet downwards; rachilla fragile. L. glumes $0.6-0.8 \mathrm{~mm}$ long, persistent, ovate, keel scaberulous, apex acute. Upper glumes $0.7-0.9 \mathrm{~mm}$ long, persistent, ovate, 1nerved, keel scaberulous, apex acute. Lemmas 1.1$1.3 \mathrm{~mm}$ long, elliptic, scaberulous, membranous, apex obtuse. Paleas $0.9-1.2 \mathrm{~mm}$ long, keels with tuberclebased hairs, apex truncate. Stamens 3; anthers ca. $0.2 \mathrm{~mm}$ long. Caryopses $0.4-0.5 \mathrm{~mm}$ long, ellipsoid, terete, pericarp finely rugulose, reddish brown.

Distribution.- North and South America, Africa, and Asia.

Ecology.- In open and dry grassland.

Vernacular.- Ya kho niao.

Notes.- This species is very similar to E. tenella but differs by having sticky glandular patches below the culm nodes. Veldkamp ${ }^{10}$ treated this name under E. amabilis which is placed here as a synonymy of E. tenella.

Specimens examined.- Khon Kaen: Khon Kaen University, Sa Plastic, 12 November 2008, W. Chaisongkram 153 (KKU). Chon Buri: Thung Brong, 13 February 1971, J.F. Maxwell 71-83 (AAU).

\section{CONCLUSIONS}

This paper provides an updated taxonomic account of Eragrostis in Thailand. A total of 23 species are recognized in Thailand including two species which are introduced, E. superba and E. tef. The majority of species occur widely in open areas and on sandy soils from low altitudes up to $2500 \mathrm{~m}$, except $E$. capensis that is strictly distributed in Phu Kradueng National Park, Loei Province. Twelve species which were previously mentioned ${ }^{8}$ to exist in Thailand: E. chloromelas, E. curvula, E. elongata, E. inamoena, E. kerrii, E. macilenta, E. minor, E. multicaulis, E. pubescens, E. subsecunda, E. trichodes, and E. udawensis were excluded. There is no specimen under these names deposited in any herbarium in Thailand or overseas. They might be wrong determinations. Comments on some nomenclatural decisions are given in the notes under each species description. The identification key constructed for Thai Eragrostis was based mainly on the spikelet disarticulation and the shape of the caryopsis, and it can be divided into 4 informal groups: the disarticulation occurring below the glumes, the disarticulation occurring from the top of the spikelet downwards, the disarticulation occurring from the base of the spikelet upwards and trigonous to reniform caryopsis, and the disarticulation occurring from the base of the spikelet upwards and ellipsoid or orbic- 
ular caryopsis. However, these characters, although useful for key construction, are artificial and should be used carefully for phylogenetic interpretation at infrageneric level.

Acknowledgements: This study was granted by the Office of the Higher Education Commission, Thailand. The first author was supported by a CHE Ph.D. scholarship. We would like to thank the Department of Biology, Faculty of Science, Khon Kaen University for facilities provided during this work. We are also indebted to the curators of the herbaria of AAU, BK, BKF, BM, C, CMU, E, G, K, KKU, $\mathrm{L}$, LINN, QBG, and TCD for providing the specimens in this study.

\section{REFERENCES}

1. Peterson PM, Romaschenko K, Johnson G (2010) A classification of the Chloridoideae (Poaceae) based on multi-gene phylogenetics tree. Mol Phylogenet Evol 55, 580-98.

2. Clayton WD, Renvoize SA (1986) Genera Graminum Grasses of the World. Her Majesty's Stationery Office, London, p 389.

3. Simon BK, Clayton WD, Harman KT, Vorontsova M, Brake I, Healy D, Alfonso Y (2011) GrassWorld, http: //grassworld.myspecies.info/.

4. Lazarides M (1980) Phanerogamarum Monographiae Tomus XII: The Tropical Grasses of Southeast Asia (Excluding Bamboos). Strauss and Cramer, Germany, p 225.

5. Peterson PM (2003) Eragrostis Wolf. In: Barkworth ME, Capels KM, Long S, Piep MB (eds.). Flora N Am 25, 65-105.

6. Wolf NM (1776) Genera Plantarum Vocabulis Characteristicis Definita. Published by the author, Königsberg.

7. Ross R (1966) The generic names published by N.M. von Wolf. Acta Bot Neerlandica 15, 147-61.

8. Nanakorn W, Norsaengsri M (2001) Species Enumeration of Thai Gramineae. Herbarium Queen Sirikit Botanic Garden, Thailand, p 92.

9. Holmgren PK, Holmgren NH (2001) Index Herbarium: A Global Directory of Public Herbaria and Associated Staff. New York Botanical Garden's Virtual Herbarium [sweetgum.nybg.org/ih/].

10. Veldkamp JF (2002) Revision of Eragrostis (Gramineae, Chloridoideae) in Malesia. Blumea 47, 157-204.

11. Lazarides M (1997) A revision of Eragrostis (Eragrostideae, Eleusininae, Poaceae) in Australia. Aust Syst Bot 10, 77-187.

12. Shouliang C, Peterson PM (2006) Eragrostis Wolf. In: Wu ZY, Raven PH, Hong DY (eds) Flora of China. Vol. 22 (Poaceae). Science Press, Beijing, and Missouri Botanical Garden Press, St. Louis, pp 471-9.

13. Vignolo F (1904) Sul valore sistematica della Poa cilianensis Ali. (1785). Malpighia 18, 380.
14. Hitchcock AS (1908) Type of American grasses: a study of the American species of grasses described by Linnaeus, Gronovius, Sloane, Swartz \& Michaux. Contrib Unit States Natl Herb 12, 121.

15. Stapf O (1897) Eragrostis Wolf. In: Hooker JD (ed) Flora of British India Vol. 7. L. Reeve Co. Ltd, The Oast house, Brook, NR. Ashford, Kent, England, pp 312-27.

16. Bor NL (1959) The Grasses of India, Burma and Ceylon Vol. 1. Pergamon Press, London, pp 495-516.

17. Scholz H (2000) Typification of Linnaean plant names in the Poaceae (Gramineae). Taxon 48, 256.

18. Mitra RL, Jain SK (1980) On the identity of four grasses in Hortus Malabaricus. In: Manilal KS (ed) Botany and History of Hortus Malabaricus. Oxford \& IBH Publishing, New Delhi, Bombay, Calcutta, India, pp 149-57.

19. Jansen P (1953) Notes on Malaysian Grasses. Reinwardtia 2,: 225-350.

20. Phillips SM (1995) Poaceae (Gramineae). In: Hedberg I, Edwards S (eds) Flora of Ethiopia and Eritrea Vol. 7. National Herbarium, Addis Ababa, Department of Systematic Botany, Upsala, p 122. 